\title{
Regulation of Synaptic Connectivity: Levels of Fasciclin II Influence Synaptic Growth in the Drosophila CNS
}

\author{
Richard A. Baines, ${ }^{1}$ Laurent Seugnet, ${ }^{2}$ Annemarie Thompson, ${ }^{2}$ Paul M. Salvaterra, ${ }^{3}$ and Michael Bate ${ }^{2}$ \\ ${ }^{1}$ Department of Biological Sciences, University of Warwick, Coventry, CV4 7AL, United Kingdom, ${ }^{2}$ Department of \\ Zoology, University of Cambridge, Cambridge, CB2 3EJ, United Kingdom, and ${ }^{3}$ Division of Neurosciences, City of Hope, \\ Beckman Research Institute, Duarte, California 91010-0269
}

Much of our understanding of synaptogenesis comes from studies that deal with the development of the neuromuscular junction (NMJ). Although well studied, it is not clear how far the NMJ represents an adequate model for the formation of synapses within the CNS. Here we investigate the role of Fasciclin II (Fas II) in the development of synapses between identified motor neurons and cholinergic interneurons in the CNS of Drosophila. Fas II is a neural cell adhesion molecule homolog that is involved in both target selection and synaptic plasticity at the NMJ in Drosophila. In this study, we show that levels of Fas II are critical determinants of synapse formation and growth in the CNS. The initial establishment of synaptic contacts between these identified neurons is seemingly independent of Fas II. The subsequent proliferation of these synaptic connections that occurs postembryonically is, in contrast, significantly re- tarded by the absence of Fas II. Although the initial formation of synaptic connectivity between these neurons is seemingly independent of Fas II, we show that their formation is, nevertheless, significantly affected by manipulations that alter the relative balance of Fas II in the presynaptic and postsynaptic neurons. Increasing expression of Fas II in either the presynaptic or postsynaptic neurons, during embryogenesis, is sufficient to disrupt the normal level of synaptic connectivity that occurs between these neurons. This effect of Fas II is isoform specific and, moreover, phenocopies the disruption to synaptic connectivity observed previously after tetanus toxin light chaindependent blockade of evoked synaptic vesicle release in these neurons.

Key words: aCC; neural activity; RP2; synaptic activity; synaptogenesis; tetanus toxin
Numerous studies suggest that cues that guide growing axons to their targets are not in themselves sufficient to generate the precise patterns of synaptic connectivity that develop between central neurons. Such studies convincingly demonstrate that there is an additional phase of activity-dependent synaptic refinement that is required to either consolidate or eliminate specific synaptic connections and produce the precision characteristic of the mature circuitry (Goodman and Shatz, 1993; Katz and Shatz, 1996). Studies of this kind have focused primarily on developing sensory systems, and the contribution of activity to the development of motor circuitry is less clear (Haverkamp, 1986; Haverkamp and Oppenheim, 1986; Baines et al., 2001).

In Drosophila, motor neurons establish a stereotyped pattern of connections with their target muscles, even in the absence of synaptic transmission (Sweeney et al., 1995). It appears therefore that, in the periphery at least, cues that guide growing axons to their targets are sufficient to ensure the proper formation of a precise pattern of connections between presynaptic and postsynaptic cells. One of the key regulators of growth and guidance of motor axons in Drosophila is Fasciclin II (Fas II), a homolog of the vertebrate neural cell adhesion molecule (NCAM) (Grenningloh et al., 1990, 1991). Fas II is expressed in all motor neurons

Received March 4, 2002; revised May 3, 2002; accepted May 9, 2002.

This study was funded by the Wellcome Trust. M.B. is a Royal Society Research Professor. We are grateful to G. Davies, C. Goodman, J. Jaynes, M. Fujioka, A. Chiba, M. Hiramoto, and H. Skaer for flies and/or antibodies. We also thank M. Day for help with electron microscopy and M. Landgraf for comments and discussion.

Correspondence should be addressed to Dr. R. A. Baines, Department of Biological Sciences, University of Warwick, Coventry, CV4 7AL, UK. E-mail: rbaines@bio.warwick.ac.uk.

Copyright (C) 2002 Society for Neuroscience $\quad 0270-6474 / 02 / 226587-09 \$ 15.00 / 0$ and promotes adhesion between their axons as they exit the CNS. In addition to axon guidance, Fas II has also been shown to have important functions at the neuromuscular junction (NMJ) itself, in which it operates to stabilize synaptic connections and to mediate activity-dependent plasticity during postembryonic life (Schuster et al., 1996a,b; Davis et al., 1997; Davis and Goodman, 1998).

Although the role of Fas II in synaptogenesis and synaptic plasticity has been intensively studied at the embryonic and larval NMJ, it is not known whether it has similar functions during the formation and elaboration of synapses in the CNS (Schuster et al. 1996a). Fas II is abundantly expressed in the embryonic, larval, and adult CNS in Drosophila and is, therefore, ideally placed to contribute to central synapse development (Grenningloh et al., 1991). More recently, a requirement for Fas II in the formation of odor memory has been demonstrated, implicating that this CAM is also central to the mechanisms of synaptic plasticity that underlie learning (Cheng et al., 2001). Because we developed recently techniques to analyze both the morphological and functional development of synaptic connections between cholinergic interneurons and identified postsynaptic motor neurons in the Drosophila CNS (Baines and Bate, 1998; Baines et al., 1999, 2001), we are now able to address directly the contribution of Fas II in the establishment of these connections. In this study, we find apparent similarities and differences between synaptogenesis centrally and at the NMJ. We show that the embryonic development of these cholinergic central synapses, like the formation of the glutamatergic NMJ, does not require Fas II. However, Fas II is required for the elaboration of these synapses during postembryonic life. Although Fas II is not required to establish synaptic connections, the normal pattern of synaptic connectivity is, nev- 
ertheless, sensitive to changes in the relative levels of expression of Fas II in presynaptic and postsynaptic neurons. In contrast to the NMJ, in which overexpression of Fas II postsynaptically leads to the stabilization of ectopic connections, in the CNS, overexpression of Fas II either presynaptic or postsynaptically causes a reduction in synaptic input to the motor neurons concerned.

\section{MATERIALS AND METHODS}

Fly stocks. Flies were fed on apple juice agar supplemented with yeast. Wild type was Oregon-R. The transgene e5GAL3M-RRK-GAL4 (RRKGAL4), which contains regulatory sequences spanning +7.9 to $+9.2 \mathrm{~kb}$ of the even-skipped locus, as well as its $3^{\prime}$ untranslated region (Fujioka et al., 1999), was used to selectively express GAL4-UAS-driven transgenes in aCC and RP2 (this GAL4 insert is identical to, but stronger expressing than, RRC-GAL4 used in previous studies) (Baines et al., 1999). Expression of RRK-GAL4 is first evident in early stage 16 embryos, which precedes the onset of synaptogenesis (Baines and Bate, 1998). GAL4 expression was selectively suppressed in aCC/RP2 using an identical RRK promoter fragment upstream of GAL80 (M. Fujioka and J. Jaynes, unpublished observation). Cha-Gal4 (19B) contains the entire (7.4 kb) promoter sequence for choline acetyltransferase (cha), the gene encoding the synthetic enzyme for $\mathrm{ACh}$ and expresses selectively in cholinergic neurons (Salvaterra and Kitamoto, 2001). 1407-GAL4 was used to express UAS transgenes throughout the entire CNS. Expression of tetanus toxin light chain (TeTxLC) (UAS-TNTG and the inactive variant TNTVIF) and Fas II were confirmed by antibody labeling (Sweeney et al., 1995; Schuster et al., 1996a). UAS-Fas II (PEST+), fasII ${ }^{e B 112}$, fasII $I^{e 76}$, fasII $^{e 86}$, fas II $^{e 93}$, and anti-Fas II were kindly provided by C. Goodman. Fas II null ( fasI $^{e B 112}$ ) larvae were identified based on their failure to stain with Fas II antibody. UAS-Fas [glycosylphosphatidylinositol (GPI)] was provided by G. Davis (University of California, San Francisco, San Francisco, CA), UAS-connectin by A. Chiba (University of Illinois, Urbana, IL), and UAS-Fas I by M. Hiramoto (University of Tokyo, Bunkyo, Japan).

Embryo dissection. Larvae were dissected, and central neurons were accessed as described by Baines and Bate (1998). The embryo was viewed using a $63 \times$ water immersion lens combined with Nomarski optics (Olympus BX50W I microscope; Olympus Optical, Tokyo, Japan).

DiI labeling and electron microscopy. Larvae were dissected, fixed in

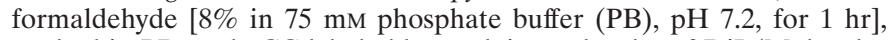
washed in $\mathrm{PB}$, and aCC labeled by applying a droplet of DiI (Molecular Probes, Eugene, OR) to its NMJ on muscle DA1. After overnight incubation at $4^{\circ} \mathrm{C}$, embryos were examined with epifluorescence, and those preparations in which only aCC was labeled were prepared for electron microscopy. Suitable embryos were fixed again in formaldehyde ( $8 \%$ and $75 \mathrm{~mm} \mathrm{~PB}, \mathrm{pH} \mathrm{7.2,} \mathrm{for} 8 \mathrm{~min}$ ), washed, and transferred into Tris buffer $(0.1 \mathrm{M}, \quad \mathrm{pH}$. 7.5) before photoconversion using 3,3'diaminobenzidine tetrachloride $(3 \mathrm{mg} / \mathrm{ml}$ in Tris buffer; Fisher, Loughborough, UK). After washing in Tris buffer followed by $\mathrm{H}_{2} \mathrm{O}$, embryos were postfixed in osmium $\left(1 \%\right.$ in $\mathrm{dH}_{2} \mathrm{O}$, for $\left.1 \mathrm{hr}\right)$, stained with aqueous uranyl acetate ( $2 \%$, for $30 \mathrm{~min})$, dehydrated, and embedded in Araldite resin. Embryos were sectioned at $2 \mu \mathrm{m}$ thickness until labeled profiles were encountered, at which point a series of ultrathin sections $(30-50$ $\mathrm{nm}$, silver-gray) were taken. Labeled neurons were not serially sectioned in their entirety but instead were sampled at $2 \mu \mathrm{m}$ intervals, with 20-30 consecutive ultrathin sections taken at each successive level. For each genotype, sections were taken from at least five neurons sectioned from at least four animals. Sections were stained with lead citrate $(5 \mathrm{~min})$ and analyzed on a Philips EM 300.

Electrophysiology. The procedure for whole-cell recordings and composition of salines used are described by Baines and Bate (1998), with the exception that potassium methylsulfonate was substituted for $\mathrm{KCl}$ in the patch saline. This substitution greatly extends the maximum recording time possible for these neurons (up to a maximum of $\sim 20 \mathrm{~min}$ ). Only cells with an input resistance $>1 \mathrm{G} \Omega$ (average of $4.05 \pm 0.46 \mathrm{G} \Omega ; n=$ 100 ; mean \pm SE) were accepted for analysis. Current traces were sampled at $20 \mathrm{kHz}$ and were filtered at $2 \mathrm{kHz}$. All recordings were made at room temperature $\left(22-24^{\circ} \mathrm{C}\right)$. Cells were unequivocally identified by labeling with Lucifer yellow (K salt, $0.1 \%$; Molecular Probes), which was included in the patch saline. Spontaneous currents were recorded in the presence of TTX (100 nM; Alomone Labs, Jerusalem, Israel).

Statistics. Data were compared using the nonparametric Mann-Whitney $U$ test, unless stated otherwise. Results were deemed significant at $p \leq 0.05$. All values shown are mean $\pm \mathrm{SE}$
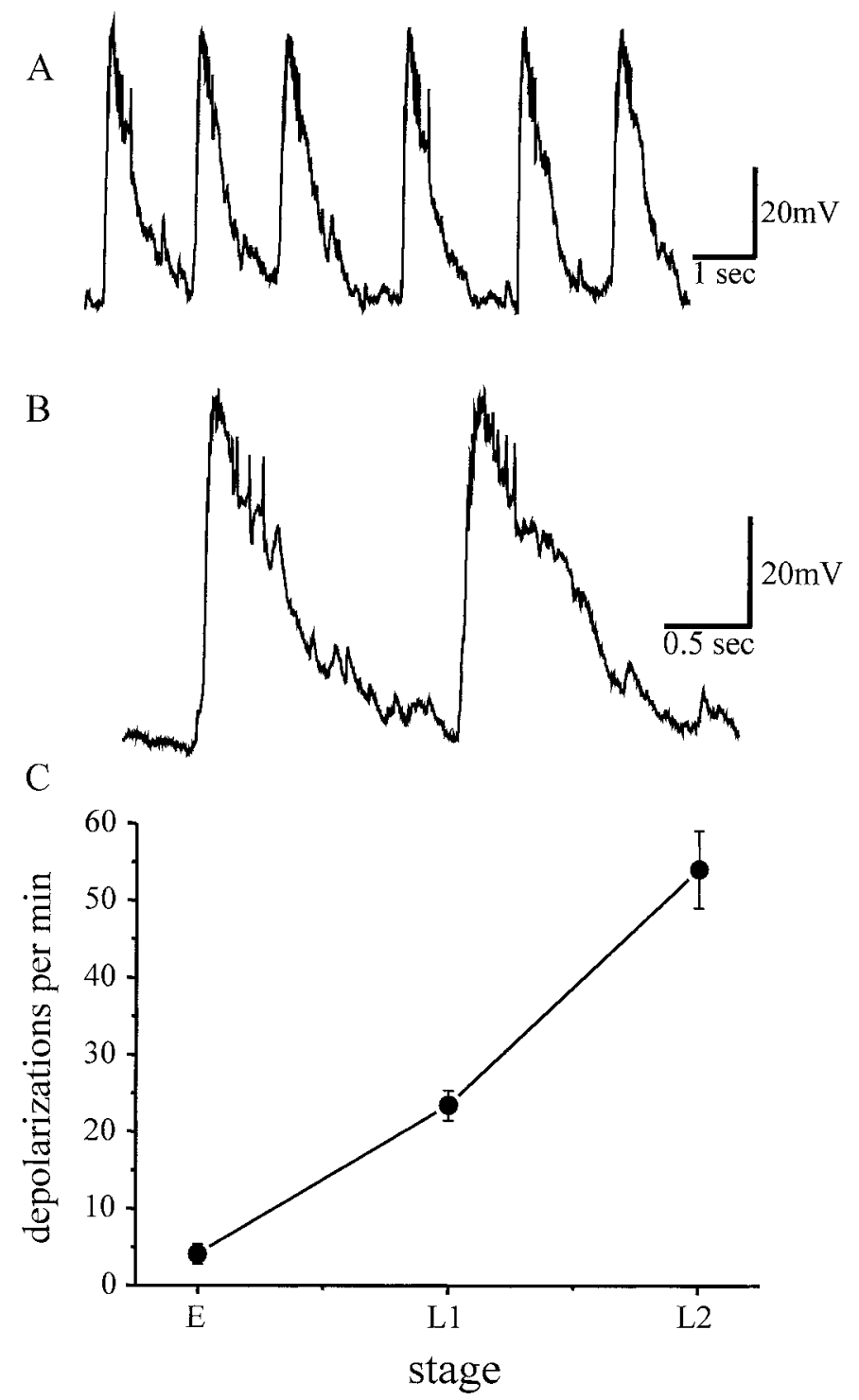

Figure 1. Synaptic drive to the aCC/RP2 motor neurons. $A, B$, Wholecell current-clamp recordings from either aCC or $\mathrm{RP} 2$ (aCC shown) in wild-type young first instar larvae show rhythmic depolarizations that are sufficient to evoke action potentials, which are more clearly visible in trace $B$. C, Synaptic depolarizations are first evident in late stage 17 embryos (labeled $E)$ and increase in frequency during larval development $(L 1$, first instar; $L 2$, second instar). Values are mean $\pm \mathrm{SE} ; n \geq 10$.

\section{RESULTS}

\section{Motor neurons receive rhythmic synaptic input from cholinergic interneurons during embryonic and larval development}

Whole-cell current-clamp recordings from two identified motor neurons, aCC and RP2, in young first instar larvae (L1, 4 hr after hatching at $25^{\circ} \mathrm{C}$ ) reveal depolarizations that are long-lived (lasting for up to $2 \mathrm{sec})$, rhythmic $(23.4 \pm 1.9$ events per min; $n=24)$, and sufficient to evoke action potentials in the motor neurons (Fig. 1). No differences were seen between these two motor neurons, and this reflects their almost identical electrophysiological properties (Baines et al., 2001). The frequency of these rhythmic depolarizations, which we term suprathreshold throughout this study to denote the fact that they elicit spikes, increases during larval development such that, in young second instar larvae (L2, 28 hr after 


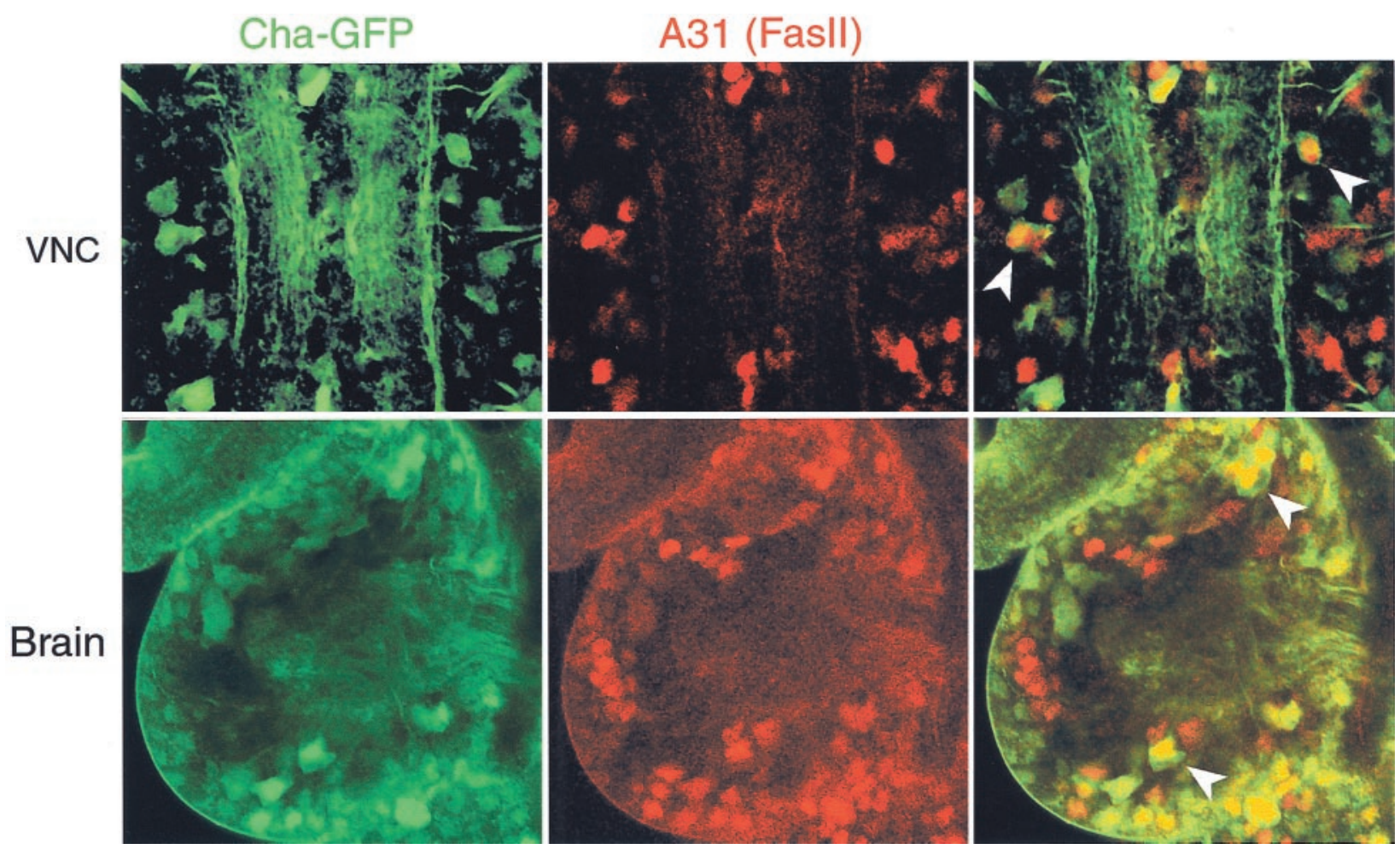

Figure 2. A subpopulation of cholinergic interneurons coexpresses Fas II. Confocal sections $(2 \mu \mathrm{m})$ through the ventral nerve cord $(V N C)$ and brain lobe (Brain) in a late stage 17 embryo. Cholinergic neurons are visualized by anti-GFP (cha B19-GAL4 driving UAS-GFP; green), and Fas II expression is reported by a $\mathrm{P}$ element driving a nuclear $\beta$-galactosidase ( $\beta$-gal antibody labeled $\mathrm{red}$ ). A number of cholinergic cells coexpress Fas II in both the ventral nerve cord and brain lobe (merged image on right, arrowheads).

hatching), the average frequency observed is $54 \pm 5$ per $\min (n=$ 7) (Fig. 1C). These depolarizations, which are first evident, although at a much lower frequency, in late stage 17 embryos ( 18-19 hr after egg laying) (Fig. 1C) (Baines et al., 1999), probably reflect the synaptic drive from presynaptic interneurons that form part of the motor pattern generator. Although we are unable to identify any individual interneurons that make synaptic contact with aCC/RP2, we established that these suprathreshold inputs are cholinergic. This conclusion is based on a number of observations. First, the presence of cholinergic antagonists blocks the synaptic inputs to aCC/RP2 (Baines et al., 1999). Second, blocking neurotransmitter release in all cholinergic neurons by expression of TeTxLC (Sweeney et al., 1995) causes embryonic paralysis and a total absence of synaptic inputs to motor neurons (R. A. Baines, unpublished data). Third, these currents are absent in cha mutants that lack acetylcholine (Baines et al., 2001). Fourth, the reversal potential of these depolarizations is $\sim 0 \mathrm{mV}$, which is identical to currents produced by iontophoretic application of ACh to these neurons (Baines, unpublished data).

\section{Fas II is expressed in a subpopulation of cholinergic interneurons}

Motor neurons, including aCC, express Fas II (Grenningloh et al., 1991; Van Vactor et al., 1993), but the extent of Fas II expression in cholinergic interneurons is not known. To determine this, we crossed a $\beta$-galactosidase enhancer trap line inserted in fasII (A31) (Ghysen and O'Kane, 1989) to flies carrying a single copy of both B19-GAL4 (which is expressed in all cholinergic neurons) (Salvaterra and Kitamoto, 2001) and UAS-GFP. The neuronal expression pattern of A31 mirrors that of fasII mRNA, and the $\mathrm{P}$ element does not alter Fas II protein expression or induce a mutant phenotype (Grenningloh et al., 1991). Confocal analysis of CNS from both early (at the initiation of synaptogenesis) and late stage 17 (mature synapses; Baines and Bate, 1998) embryos shows that a proportion of cholinergic neurons (labeled green) also express $\beta$-galactosidase (Fig. 2 , red). The number of coexpressing neurons is difficult to determine precisely but is relatively small. There are between 5 and 10 such neurons per segment in the ventral nerve cord (Fig. $2 A$ ) and between 20 and 30 in each brain lobe (Fig. 2B). Thus, in addition to motor neurons, a subpopulation of cholinergic interneurons appear to express fasII, although we cannot, of course, infer from this that these particular interneurons are presynaptic to aCC/RP2.

\section{Fas II is not required for central synaptogenesis}

Fas II is not required for the formation of the embryonic NMJ, although it is required later for the maintenance and proliferation of these synaptic contacts (Schuster et al., 1996a,b). The requirement for Fas II for plastic changes at the NMJ was established, in part, through the use of a series of fasII alleles. These alleles, which affect only the level of Fas II expression but not protein structure or tissue distribution, include fas $I I^{e B 112}$ (a protein null), fasII $^{e 76}$ (5-10\% of normal levels), fasII ${ }^{e 86}$ ( $\left.\sim 50 \%\right)$, and fasII $I^{e 93}$ (100\% and a control for genetic background) (Grenningloh et al., 1991; Schuster et al., 1996a). To show whether Fas II is required for the formation of a normal pattern of presynaptic inputs onto embryonic motor neurons, we studied synaptic communication in these differing fasII alleles. We analyzed synaptic drive to aCC/ RP2 and made an ultrastructural survey of connections between $\mathrm{aCC}$ and its presynaptic partner neurons. 
A
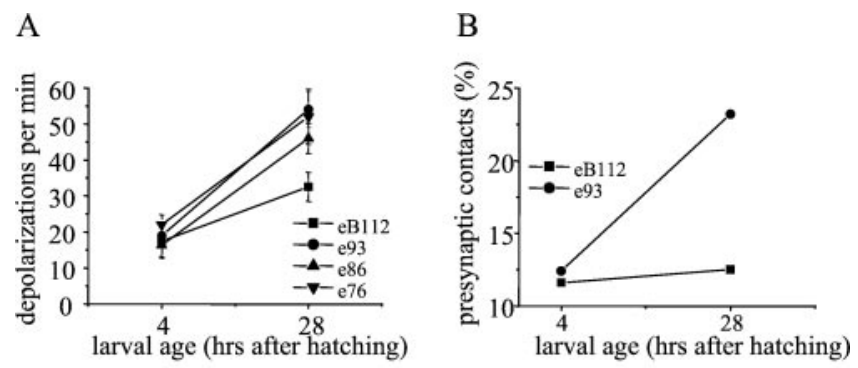

C FasI ${ }^{e 93}$ (normal)

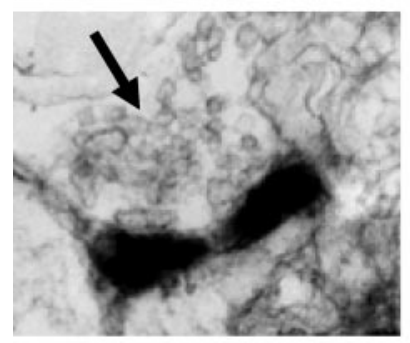

E FasII ${ }^{93}$ (control)

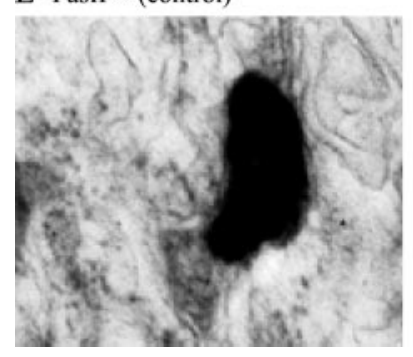

D FasI[ ${ }^{\mathrm{B} 112}$ (null)

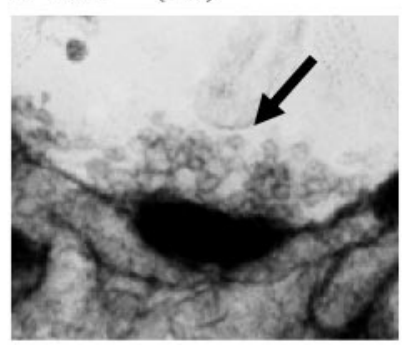

Figure 3. Synaptic proliferation during larval development requires Fas II. $A$, During the first $28 \mathrm{hr}$ of larval life, the frequency of suprathreshold synaptic inputs recorded in aCC/RP2 increases approximately twofold (control line, $\left.f a s^{e 93}\right)$. In the absence of Fas II $\left(f^{e s^{e B 112}}\right)$, this developmental increase is significantly reduced $(p \leq 0.01)$. The increase remains normal, however, in Fas II hypomorphs (lines fas ${ }^{886}$ and fas $^{776}$, respectively). Values are mean $\pm \mathrm{SE} ; n \geq 8$. $B$, Ultrastructural analysis reveals that the number of presynaptic terminals that contact aCC (see Materials and Methods) increases during the same period $\left(\mathrm{fas}^{e 93}\right)$. In the absence of Fas II $\left(\right.$ fas $\left.^{e B 112}\right)$, however, this increase fails to occur. $C, D$, In $28 \mathrm{hr}$ larvae, the presynaptic terminals (arrows) seen to contact aCC are qualitatively similar, regardless of the presence $(C)$ or absence $(D)$ of Fas II. $E$, An example of a labeled profile of aCC that is not associated with a presynaptic input.

We find that the synaptic drive to aCC/RP2 in L1 is not significantly different in these alleles (Fig. $3 A$ ). The frequency of suprathreshold synaptic inputs recorded was $17.4 \pm 4.6$ ( fasII ${ }^{e B 112}$ ), $22 \pm 2.8\left(\right.$ fasI $\left.^{e 76}\right), 16.3 \pm 3.2\left(\right.$ fasI $\left.^{e 86}\right)$, and $19 \pm 2\left(\right.$ fasII $\left.^{e 93}\right)(n \geq$ $8 ; p>0.05)$. Dendritic processes in the neuropil belonging to aCC were revealed by photoconversion of DiI-labeled neurons to produce an electron-dense precipitate. Sites of presynaptic input were identified by the presence of a cluster of clear synaptic vesicles, with a requirement that some vesicles be docked to the presynaptic membrane immediately adjacent to the labeled profile (Fig. 3C,D) (Baines et al., 1999, 2001). There was no significant difference in the number of presynaptic terminals that contact aCC in L1 in the presence or absence of Fas II (12.4\%, 32 of 258 labeled profiles examined exhibited a presynaptic element, fas $I I^{e 93}$ vs $11.6 \%, 32$ of 276 , fasII ${ }^{e B 112} ; p>0.05 ; \chi^{2}$ test) (Fig. $3 B$ ). We conclude that Fas II is not required for the establishment of synaptic connections

between embryonic motor neurons and their presynaptic partners.

\section{Fas II is required for postembryonic synaptic proliferation}

During the first $28 \mathrm{hr}$ of larval life, the frequency of suprathreshold synaptic drive to aCC/RP2 increases considerably $(19 \pm 2.0$ to $54 \pm 5$ events per min, fasII ${ }^{e 93}$ ) (Fig. $3 A$ ). This change is associated with a similarly large increase in the number of presynaptic terminals that contact aCC $\left(23.2 \%, 63\right.$ of 271 , fasII $\left.I^{e 93}\right)$ (Fig. $3 B$ ). This suggests that, in addition to a proliferation of synaptic connections between presynaptic neurons and aCC/RP2, the probability of firing activity in the presynaptic neurons also increases during larval development. In the absence of Fas II, the frequency of synaptic drive to aCC/RP2 still increases during this period (increasing to $32.5 \pm 4$ events per min; $n=8$; fasII ${ }^{e B 112}$ ), although this increase is significantly less than when Fas II is present ( $p \leq 0.01$ ) (Fig. 3A). Strikingly, however, in the absence of Fas II, the number of presynaptic terminals that contact aCC remains unchanged $(12.5 \%, 34$ of 272$)$ (Fig. $3 B)$. The ultrastructure of the presynaptic terminals at this stage is qualitatively indistinguishable from those that develop in WT (Fig. 3C,D). Thus, although there is no absolute requirement for Fas II to support either initial synapse formation or the consolidation of these early connections, it is required for the proliferation of these contacts postembryonically. The increase in the frequency of synaptic drive to aCC/RP2 is not affected in the hypomorphic alleles examined $\left(52 \pm 7.6\right.$, fas $I I^{e 76}$ and $46 \pm 4.2$, fas II $\left.I^{e 86} ; n \geq 8\right)$ (Fig. 3A), suggesting that even severely reduced levels of Fas II are still sufficient to allow normal postembryonic maturation of the motor network.

\section{Genetic manipulations that increase the levels of Fas II in motor neurons reduce synaptic input}

Although the embryonic NMJ forms normally in the absence of Fas II, an increase in Fas II (PEST + ) expression in the postsynaptic muscle cell during synaptogenesis is sufficient to alter the pattern of synaptic connectivity by driving the formation of additional, ectopic synaptic contacts by innervating motor neurons (Davis et al., 1997). We decided to investigate whether increased levels of Fas II expressed postsynaptically in motor neurons have a similar effect on synapse formation in the CNS. Three major isoforms of Fas II have been reported in Drosophila (Lin et al., 1994; Goodman et al., 1997). Two of these contain a single transmembrane (TM) domain and are distinguishable by the presence of a PEST degradation sequence (PEST + ) (Rechsteiner, 1988) in the cytoplasmic domain of one but not in the other (PEST-). The third isoform is a GPI-linked form that lacks a TM domain. Using current clamp, we measured the effect of increasing both Fas II (PEST+) and Fas II (GPI-linked) in $\mathrm{aCC} / \mathrm{RP} 2$ by recording the frequency of suprathreshold synaptic inputs observed in these neurons.

Expressing Fas II (PEST+) in aCC/RP2 (using an RRKGAL4 driver) significantly reduced the frequency of suprathreshold inputs recorded in L1 $(7.2 \pm 1.8$ vs $26 \pm 3.3$ events per min; $n=10 ; p \leq 0.001$ ) (Fig. $4 A$ ). The effect of expressing Fas II in $\mathrm{aCC} / \mathrm{RP} 2$ was similar for a second independent UAS insert (third chromosome, $10.5 \pm 3.2$ vs $24 \pm 3.0$ events per $\min ; n=9 ; p \leq$ 0.01 ) and was rescued by the simultaneous coexpression of a GAL4 inhibitor (RRK-GAL80) in aCC/RP2 (20 \pm 4 events per $\min ; n=8 ; p>0.05$ ) (Fig. $4 A$ ). Together, these controls rule out any contribution of genetic background. In addition to recording 
A aCC/RP2 (FasIl expression)

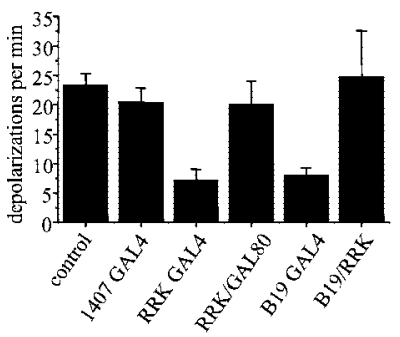

C aCC/RP2 (FasII expression)

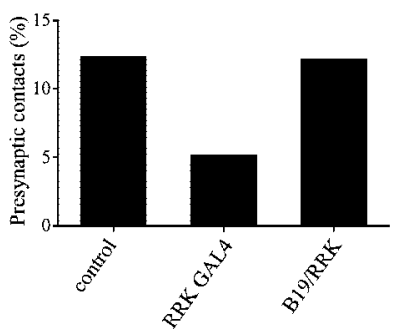

B RP3 (FasII expression)

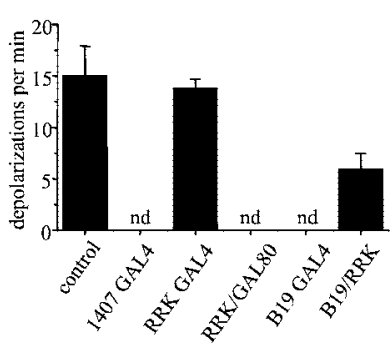

D RP3 (FasII expression)

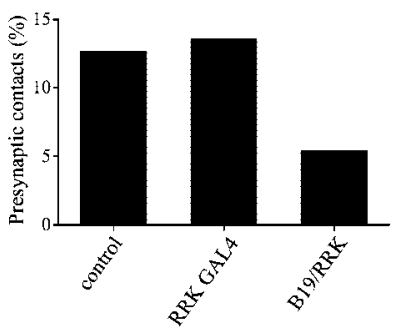

Figure 4. Increased levels of Fas II in either the presynaptic or postsynaptic neurons disrupt synaptogenesis. $A$ shows the frequency of suprathreshold synaptic inputs recorded in aCC/RP2 in L1. control represents the average frequency seen in parental GAL4s (1407, RRK, and B19) and UAS transgenic lines (no individual line was significantly different from any other). Expression of Fas II (PEST + ) in all neurons of the CNS (1407-GAL4) does not influence the frequency of synaptic inputs. However, selective expression of Fas II in either aCC/RP2 (RRK-GAL4) or presynaptic cholinergic (B19-GAL4) neurons significantly reduces input frequency ( $p \leq 0.001$ for both treatments). Simultaneous expression of Fas II in both presynaptic cholinergic neurons and aCC/RP2 (B19/RRKGAL4s) does not reduce the frequency of synaptic drive. The effect of expressing Fas II in aCC/RP2 is rescued by the presence of GAL80 in these motor neurons (RRK-GAL4/GAL80). For all values, $n \geq 8$; mean \pm SE. $B$, Expression of Fas II (PEST+) in aCC/RP2 (RRK-GAL4) does not influence the frequency of suprathreshold synaptic drive recorded in the RP3 motor neuron (which does not express GAL4 in these larvae). However, combined expression in both aCC/RP2 and cholinergic interneurons (B19/RRK-GAL4s) results in a significant decrease $(p \leq 0.05$; for explanation, see Results). $C$, Ultrastructural analysis reveals that expression of Fas II (PEST + ) in aCC (RRK-GAL4) significantly reduces the number of presynaptic terminals observed to contact this neuron $(p \leq$ $0.05 ; \chi^{2}$ test). Simultaneous expression of Fas II in both aCC and cholinergic neurons (B19/RRK) does not, however, affect the number of presynaptic terminals that contact aCC. $D$, Expression of Fas II (PEST +) in aCC does not reduce the number of presynaptic terminals that contact RP3. Presynaptic terminals contacting this neuron (which does not express GAL4 in these larvae) are significantly reduced in number after the combined expression of Fas II in both aCC/RP2 and cholinergic neurons $\left(p \leq 0.05 ; \chi^{2}\right.$ test $)$.

from aCC/RP2, we recorded the synaptic drive to a third, control motor neuron (RP3) that does not express GAL4 in these embryos and larvae. The frequency of suprathreshold inputs in RP3 was unaffected by expression of Fas II in aCC/RP2 $(15 \pm 2.9$ vs $13.8 \pm 0.9$ events per min, experimental vs control; $n=5 ; p>$ 0.05) (Fig. 4B). Expressing the GPI-linked isoform of Fas II in aCC/RP2 did not significantly reduce the frequency of suprathreshold synaptic drive $(19.1 \pm 3.9$ events per min; $n=5 ; p>$ 0.05). Expression of Fas I or connectin, two other Drosophila CAMs (Chiba, 1999), in aCC/RP2 also failed to affect the frequency of synaptic input $(23.2 \pm 3.5$ and $23 \pm 3.6$ events per min, respectively; $n=5 ; p>0.05)$. We conclude that the observed reduction in suprathreshold inputs from presynaptic partner neurons is a consequence specifically of enhancing Fas II (PEST+) expression in aCC/RP2.
To analyze more rigorously the effect that increasing expression of Fas II (PEST+, hereinafter termed Fas II) in aCC/RP2 has on the strength of synaptic currents, we repeated the recordings in voltage clamp. Voltage clamp is advantageous because it removes any contributions of voltage-gated ion channels in the motor neuron membrane to the synaptic currents observed. This is of particular relevance because our previous work shows that, when deprived of excitatory synaptic input, aCC/RP2 compensate by upregulating the relative strength of specific voltage-gated channels (Baines et al., 2001). Recordings of synaptic transmission, under voltage clamp $\left(V_{\mathrm{h}}-60 \mathrm{mV}\right)$, show clearly that, when Fas II is expressed in aCC/RP2, the amplitude distribution of evoked synaptic currents is significantly reduced compared with controls (Fig. 5Ai,Aii). Thus, the underlying effect of overexpressing Fas II in aCC/RP2 appears to be one of reduced synaptic function, which results in a significant reduction of the suprathreshold synaptic drive that these neurons receive. Such a shift in distribution could result from either a reduction in the total number of presynaptic terminals that normally excite these neurons or, alternatively, from a more general weakening of the synaptic efficacy of the normal complement of synapses present. Because we do not know whether evoked currents in these neurons result from activity at just one synapse or require the combined activity of many synapses, we cannot readily distinguish between these different mechanisms. However, an analysis of spontaneous currents in aCC/RP2 shows no such reduction in amplitude distribution after expression of Fas II in these neurons (Fig. 5Bi,Bii). Moreover, the frequency of spontaneous events is significantly reduced after expression of Fas II $(11.1 \pm 2.7$ vs $4.75 \pm 0.6$ events per min, control vs Fas II expression; $p<0.05)$. A reduction in spontaneous current frequency, without an associated change in current amplitude, is consistent with a reduction in the number of presynaptic input sites to aCC/RP2 rather than a more general weakening of the synapses normally present (see below).

\section{Synaptic transmission is influenced by the relative levels of Fas II expression across the synapse}

The fact that genetic manipulations that increase the levels of Fas II expression in motor neurons are sufficient to alter the synaptic drive that they receive suggests that synaptogenesis might be sensitive to the relative levels of Fas II expression in the presynaptic and postsynaptic neurons. To test this idea, we used a GAL4 construct driven by the Drosophila cha promoter that is expressed in all cholinergic neurons (B19-GAL4) (Salvaterra and Kitamoto, 2001). Using this construct to drive Fas II expression in cholinergic neurons, we found a significant reduction in the frequency of suprathreshold synaptic drive recorded in aCC/RP2 $(8 \pm 1.2$ vs $24 \pm 3$ events per min; $n=10 ; p \leq 0.001$ ) (Fig. $4 A$ ). Thus, increased Fas II in either the presynaptic or postsynaptic neurons is seemingly sufficient to disrupt synaptogenesis.

It might be argued that misexpressing Fas II interferes with synaptogenesis by altering axon guidance before synapse formation. However, this was a possibility that we tested by an additional experiment in which we expressed Fas II simultaneously in both cholinergic neurons and in aCC/RP2 (RRK/B19-GAL4). Recordings from aCC/RP2 in $\mathrm{L} 1$ of this genotype show no significant reduction in the frequency of suprathreshold synaptic inputs $(24.7 \pm 7.9$ events per $\min ; n=5)$ (Fig. $4 A)$. In this experiment, however, recordings from the RP3 motor neuron showed a marked reduction in synaptic drive $(6 \pm 1.5$ vs $15 \pm 2.9$ events per $\min ; n=5$; $p \leq 0.01$ ) (Fig. $4 B$ ). Thus, although we 


\section{evoked currents}
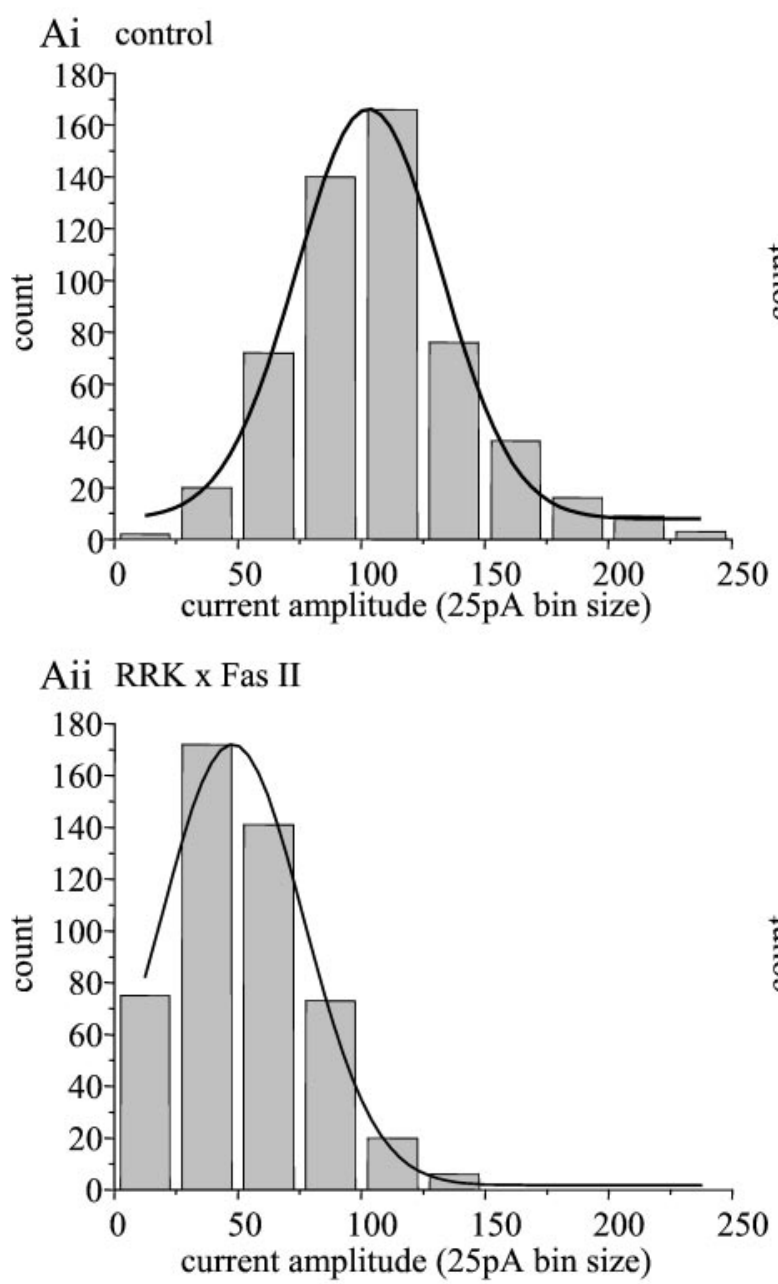

\section{spontaneous currents}

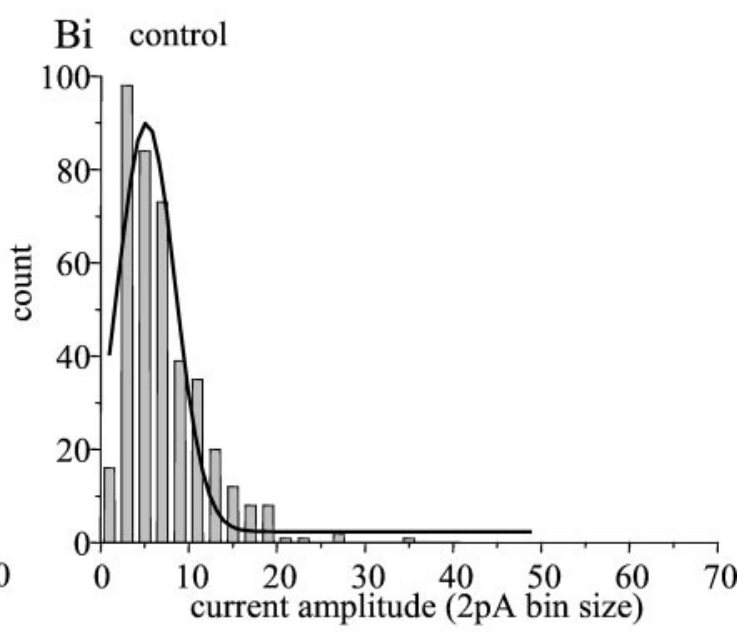

Bii RRK x Fas II

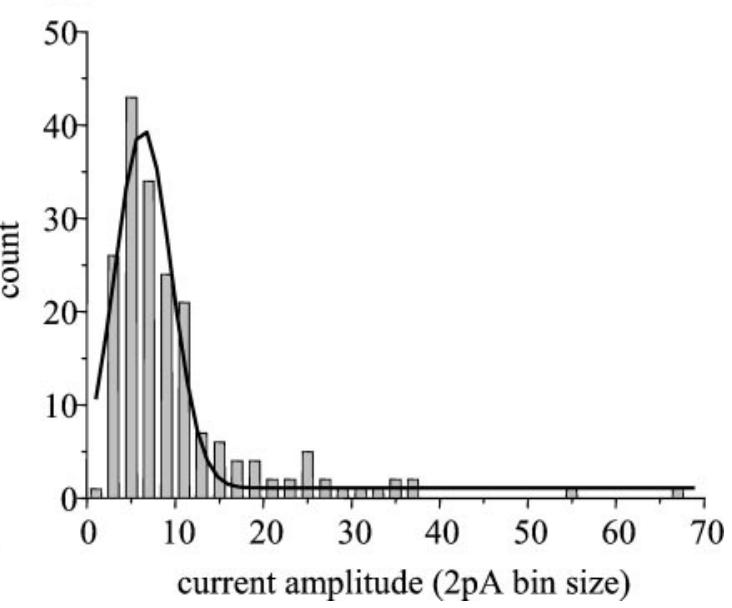

Figure 5. Fas II expression in aCC/RP2 reduces the strength of synaptic inputs. Ai, Amplitude distribution of evoked synaptic currents recorded in voltage clamp $\left(V_{\mathrm{h}}-60 \mathrm{mV}\right)$ from aCC/RP2 in control L1 (GAL4 and UAS parental lines). Current amplitude is normally (Gaussian) distributed with a peak amplitude of $-102 \pm 1.5 \mathrm{pA}(n=542)$. Aii, The amplitude distribution of synaptic currents recorded in aCC/RP2 is significantly reduced after expression of Fas II (PEST +$)$ in just these neurons $(p<0.001)$. Under these conditions, the distribution is centered around a mean amplitude of $-48 \pm$ $1.3 \mathrm{pA}(n=487)$. $B$, Spontaneous currents (those which persist in the presence of TTX) show no significant difference in amplitude distribution attributable to expression of Fas II in aCC/RP2. In control L1 (Bi; GAL4 and UAS parental lines), currents are normally distributed around a mean of $-5.2 \pm 0.3 \mathrm{pA}(n=398)$, whereas after expression of Fas II in aCC/RP2 (Bii), the distribution mean is $-6.3 \pm 0.2 \mathrm{pA}(n=190)$. Individual currents were obtained from at least six neurons for each analysis.

have not shown directly that this manipulation does not affect axon guidance, the experimental outcome reinforces our view that an imbalance in presynaptic and postsynaptic levels of Fas II expression interferes with synaptogenesis, because in this experiment, RP3, which also receives input from cholinergic presynaptic neurons, does not itself express GAL4. Thus, in the case of RP3, elevated expression of Fas II is only presynaptic. We also expressed Fas II in all neurons of the embryo (1407-GAL4) but, again, did not observe any alteration in the frequency of suprathreshold synaptic currents recorded in aCC/RP2 in L1 $(20 \pm 2.5 \mathrm{vs}$ $24.7 \pm 8$ events per min, respectively, experimental vs control; $p>0.05$ ) (Fig. 4A). We conclude from these electrophysiological data that the normal development of synaptic communication between embryonic motor neurons and their presynaptic partners is sensitive to the relative amount of Fas II expressed on either side of the synapse but is unaffected by the absolute level of Fas II present.

\section{Synaptogenesis is disrupted by a forced imbalance of Fas II between synaptic partner neurons}

We showed that experimentally induced increases in levels of Fas II, in either presynaptic or postsynaptic neurons, are sufficient to reduce synaptic efficacy. However, the mechanism that underlies this effect is unknown. Our electrophysiological analysis (see above) is consistent with overexpression of Fas II resulting in a reduction in the number of synapses that normally form between these populations of neurons. Because we are able to quantify the number and appearance of presynaptic terminals that contact $\mathrm{aCC} / \mathrm{RP} 2$, this hypothesis is testable.

An ultrastructural analysis reveals that increased expression of Fas II in aCC (RRK-GAL4) is accompanied by a significant reduction in the number of presynaptic terminals that contact this neuron in L1 (5.2\%, 32 of 258 vs $12.4 \%, 17$ of $324 ; p \leq 0.05 ; \chi^{2}$ test) (Fig. 4C, RP2 was not examined). The expression of Fas II 
in these larvae does not, however, affect the number of presynaptic terminals that contact RP3, the control neuron that does not express GAL4 (12.7\%, 40 of 314 vs 13.6\%, 41 of 302 profiles, control vs experimental; $p>0.05$ ) (Fig. $4 D$ ). Expressing Fas II simultaneously in both cholinergic neurons and aCC/RP2 (RRK/ B19-GAL4s), which does not affect synaptic drive (see above), also has no affect on the number of presynaptic terminals that contact aCC $(12.4 \%, 32$ of 258 vs $12.2 \%, 33$ of 270 , control vs experimental) (Fig. 4C). In these same larvae, however, the number of presynaptic terminals that contact RP3 (which has a reduced frequency of synaptic input; see above), is significantly reduced $\left(5.4 \%, 15\right.$ of 277 vs $12.7 \%, 40$ of $314 ; p \leq 0.05 ; \chi^{2}$ test) (Fig. 4D). The most parsimonious explanation for these observations is that synaptogenesis between motor neurons and their presynaptic partners is decisively influenced by the relative levels of Fas II expression between the two types of neurons.

\section{TeTxLC-dependent disruption of synaptogenesis is partly dependent on Fas II}

There is a striking similarity between the results of these experiments and the effects we reported previously of misexpressing TeTxLC in the developing CNS (Baines et al., 1999). In those experiments, we showed that the selective expression of TeTxLC in $\mathrm{aCC} / \mathrm{RP} 2$ results in a reduction in synaptic input to these neurons that is comparable with the effects we describe here. The underlying cause of the reduction is, again, a failure of synapse formation between presynaptic elements and the affected motor neurons. The similarity between the results of the two experiments suggests that Fas II might be a downstream effector of TeTxLC expression. Indeed, evidence for such a link has been reported recently in the Drosophila eye in which expression of TeTxLC in photoreceptors results in enhanced levels of Fas II in these cells (Hiesinger et al., 1999).

To establish a link between the two sets of experiments, we examined the effect of TeTxLC expression in a Fas II null background $\left(\right.$ fasII $I^{e B 112}$ ). In the presence of Fas II, expression of TeTxLC in aCC/RP2 severely reduces the frequency of suprathreshold inputs to these neurons when compared with controls $(4.4 \pm 1.7$ vs $17.4 \pm 4.4$ inputs per min; mean $\pm \mathrm{SE} ; n=7 ; p \leq$ 0.01) (Baines et al., 1999). In the absence of Fas II ( fasII $\left.{ }^{e B 112}\right)$, the effect of TeTxLC expression on the frequency of such synaptic inputs is significantly diminished $(10.9 \pm 2.4$ inputs per minute; mean $\pm \mathrm{SE} ; n \geq 8 ; p \leq 0.05$ compared with TeTxLC in the presence of Fas II) (Fig. 6). This suggests that part of the effect of misexpressing TeTxLC is likely to be caused by altered levels of Fas II expression. However, the fact that the rescue of the toxin effect is incomplete in the absence of Fas II $(10.9 \pm 2.4$ vs $17.4 \pm 4.4)$ implies that TeTxLC also acts to alter the expression levels of other proteins that are involved in the formation of appropriate synaptic connectivity (adult photoreceptors; cf. Hiesinger et al., 1999).

\section{DISCUSSION}

Previous studies of Fas II and synaptogenesis have focused on the accessible synapse formed at the larval NMJ in Drosophila. In this paper, we extend these studies to the more complex issue of the formation of central synapses, using a relatively well defined set of synaptic contacts that form during embryogenesis between cholinergic interneurons and identified motor neurons. The analysis at the NMJ shows that Fas II is expressed both presynaptic and postsynaptically but that it is not required for the formation of synaptic connections between motor neurons and their target

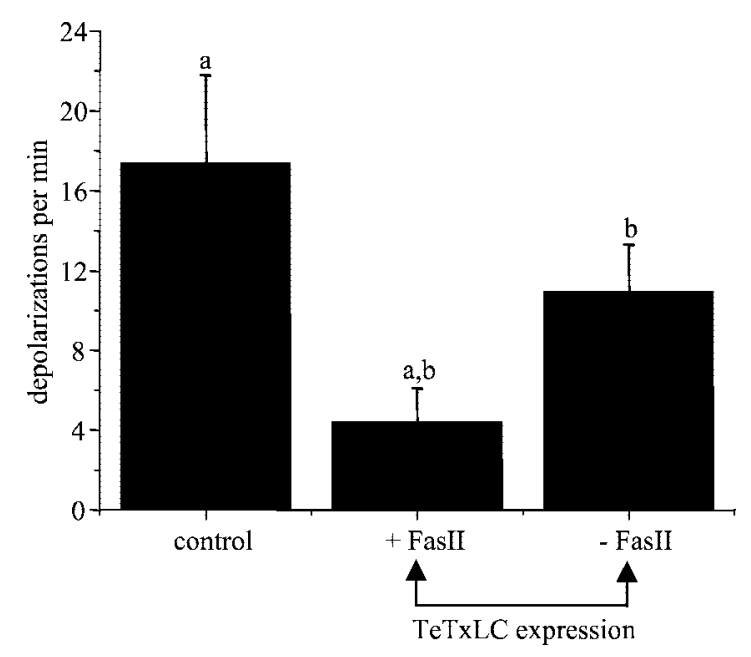

Figure 6. Fas II is required for TeTxLC-induced reduction in synaptic inputs. Expression of TeTxLC in aCC/RP2 (RRK-GAL4) is sufficient to reduce the frequency of suprathreshold synaptic inputs to these neurons (Baines et al., 1999). However, in the absence of Fas II (fasII ${ }^{e B 112}$ ), the TeTxLC-induced reduction of input is diminished. For all values, $n \geq 7$; mean \pm SE. Similarity of letters denotes statistical significance at $p \leq 0.05$. Control larvae contained either the GAL4 or UAS transgenes (but not both) and normal Fas II levels.

muscles (Schuster et al., 1996a,b). However, if Fas II is overexpressed in muscles during a critical period of embryogenesis, it allows additional, ectopic synapses to form and become stabilized on the muscles concerned (Davis and Goodman, 1998). These findings together with immunocytochemical studies of Fas II expression have suggested that, during the initial phase of synaptogenesis, Fas II is present in limiting amounts on the postsynaptic cell and that the protein then becomes aggregated under contacts formed by the innervating motor neuron, so inhibiting the formation of stable, ectopic contacts by other neurons (Davis et al., 1997). These observations imply that, although not essential to synaptogenesis, Fas II can act as a powerful determinant of the distribution and number of contacts on the postsynaptic cell.

Our first aim was to show whether or not Fas II can act in a similar manner during the embryonic formation of central synapses. We know that Fas II is expressed in motor neurons (Grenningloh et al., 1991; Van Vactor et al., 1993), and our studies show that it is also expressed in a subset of cholinergic interneurons, although we are unable to say whether these are immediately presynaptic to the motor neurons that we are studying. Moreover, we do not know the precise distribution of Fas II protein in either the presynaptic or postsynaptic neurons, nor do we know anything about the relative expression of Fas II in different neurons. However, the results of both our physiological and ultrastructural analyses show that an apparently normal pattern of interneuron to motor neuron synapses develops in the absence of Fas II. In the continued absence of Fas II, however, these synapses clearly fail to proliferate, and, as a consequence, the synaptic drive to motor neurons is reduced. On the other hand, increased Fas II expression in either the presynaptic or postsynaptic cells is sufficient to reduce synaptic inputs to the motor neurons as judged physiologically or ultrastructurally. The puzzling aspect of this latter result is that, although it suggests that, as at the NMJ, Fas II can act centrally to influence the pattern of synaptic contacts, it appears to do so in completely the opposite sense: additional Fas II reduces the number of synapses rather than promoting the for- 
mation of additional, ectopic contacts. Our findings also differ from the observed consequence of disproportionately increasing the mammalian homolog of Fas II, NCAM, in postsynaptic hippocampal neurons maintained in culture, which, similar to the NMJ, is also sufficient to strengthen synaptic connectivity (Dityatev et al., 2000). We cannot discount the possibility that increased expression of Fas II in aCC/RP2 results in the additional formation of inappropriate synaptic connections to these neurons, which may be sufficient to weaken, structurally or functionally, the connections that normally form between these neurons and their normal presynaptic partners. However, because we see a clear reduction in the number of presynaptic input sites in young first instar larvae, under these conditions, it would suggest that any such inappropriate connections are likely to have retracted by this stage. Simply interpreted, the effects that we report suggest that, although Fas II is required for postembryonic synapse proliferation, disproportionate increases in levels of Fas II in central neurons has a potentially repressive effect on the formation of synapses between the cell concerned and its putative synaptic partners, regardless of its site of expression. We should be cautious, however, in finally adopting this conclusion, because the environment of dendritic arborizations in the embryonic neuropil is likely to be complex, and we do not understand the contribution of Fas II to dendritic patterning. Thus, although increased levels of Fas II expression, or absence of this CAM, does not alter the gross morphology of aCC based on an analysis of DiI-labeled cells (Baines, personal observation), such manipulations could conceivably alter more subtle aspects of dendritic morphology and disrupt the normal pattern of synaptic connectivity. A detailed analysis of dendritic patterning in these neurons is reliant on, and must wait until, we are able to visualize individual presynaptic partner neurons.

Our experiments concentrate on two motor neurons, aCC and RP2, that innervate dorsal muscles. These neurons are identifiable in the embryonic and larval CNS and are relatively accessible to patch-clamp electrodes. In addition, the RRK-Gal4 line allows us to misexpress proteins such as Fas II selectively in these cells (Baines et al., 1999). We also monitor the results of our experiments in a third, control motor neuron, RP3, that innervates ventral longitudinal muscles. The effects of our experiments on RP3 are interesting and revealing. First, under control conditions, the frequency of suprathreshold synaptic input to RP3 is approximately one-half that seen in aCC/RP2. This suggests that, under the conditions of our experiments, RP3 (ventral muscles) and aCC/RP2 (dorsal muscles) receive distinct inputs from interneurons involved in generating rhythmic motor outputs. Second, the frequency of input to RP3 remains unchanged when the level of Fas II is increased in aCC/RP2, and the frequency of synaptic input declines in these neurons. This result suggests that the alterations in synaptic communication that we detect are the result of local events in the neurons concerned. The third observation is the most significant and reinforces our interpretation that the effects we describe depend on the relative levels of Fas II expressed in presynaptic and postsynaptic neurons. Thus, in experiments in which Fas II is simultaneously expressed in cholinergic interneurons and $\mathrm{aCC} / \mathrm{RP} 2$, the decline in input frequency to aCC/RP2, seen when Fas II is expressed in either of these sets of neurons alone, fails to occur. This result implies that it is the balance of Fas II in presynaptic and postsynaptic cells that is decisive for the formation of a normal pattern of synaptic inputs. Significantly, in this experiment, the control neuron RP3, with normal levels of Fas II, is innervated by interneurons whose level of Fas II has been increased: we predict that synaptic communication should be weakened, and this is indeed the effect that we observe. Thus, alterations in the relative levels of Fas II in presynaptic and postsynaptic cells have local effects that are selective and predictable for individual neurons. This strongly suggests that, during synaptogenesis, the balance of Fas II in presynaptic and postsynaptic cells can influence the formation of a normal pattern of synaptic contacts.

The strikingly similar results of misexpressing Fas II or TeTxLC in aCC/RP2 suggested to us that the effects of TeTxLC might be caused at least in part by elevated levels of Fas II in the neurons in which it is expressed. Indeed, we find that the toxin effects are partially rescued by the complete loss of Fas II function. This, together with our observation that an imbalance in presynaptic and postsynaptic levels of Fas II expression is sufficient to interfere with normal synaptogenesis, offers an explanation for our previously puzzling finding that blocking vesicle release from the postsynaptic neuron leads to a reduction in presynaptic input to that cell (Baines et al., 1999). If, as in photoreceptor cells, expression of TeTxLC leads to an overall increase in levels of Fas II in the affected cells (Hiesinger et al., 1999), then we would expect synaptic inputs to those cells to be disturbed. The finding that the local balance of Fas II influences the formation of central synapses, together with the strong implication that alterations in vesicle trafficking can interfere with this balance, is important for our understanding of normal synaptogenesis and its control. We would predict that, although synaptogenesis can proceed successfully in the absence of Fas II, any, possibly activity-dependent, modulation of Fas II levels in presynaptic or postsynaptic cells has the potential to influence the number and pattern of connections formed in a normal embryo. How activity might regulate levels of Fas II in synaptic terminals remains to be determined. Synaptically targeted membrane proteins, including neurotransmitter receptors, are thought to be constantly moving in to and out of the synaptic membrane, this movement being dependent on successive rounds of vesicular endocytosis and exocytosis (Turrigiano, 2000). A perturbation at any point in this cycle has the potential to result in an inappropriate surface expression of these proteins and perhaps provide a viable route to influence synaptic plasticity. An example of such a mechanism is long-term sensitization in Aplysia, which involves an activity-dependent downregulation of apCAM (a homolog of Fas II) in the presynaptic sensory neuron (Mayford et al., 1992; Zhu et al., 1995). This downregulation appears to be attributable to a cAMP-dependent reduction in gene expression and a simultaneous increase in the rate of endocytotic internalization of preexisting protein from the presynaptic membrane (Bailey et al., 1992; Mayford et al., 1992).

Although Fas II is not required for the initial formation of an appropriate pattern of synaptic contacts either peripherally (at the NMJ) or centrally, our experiments show that, as at the NMJ, Fas II is essential for the further growth and elaboration of synaptic contacts during postembryonic life. During this larval phase of active feeding and growth, the increasing size of the muscles is matched by an increase in the size and complexity of motor neuron dendritic arbors (Uhler, 2001). Our ultrastructural analysis of synaptic inputs to motor neuron arbors during early larval life shows that, at least in these early phases, there is a corresponding increase in the number of presynaptic contacts on the dendrites of aCC/RP2. Strikingly, this increase fails completely in the Fas II null larvae. It is likely that this, together with the previously documented reduced innervation at the NMJ, 
contributes to the increasing sluggishness and ultimate death of these mutant larvae. We have not surveyed the presynaptic contacts formed postembryonically in the series of hypomorphic Fas II alleles, but, given the relatively normal maturation of the synaptic drive that we detect in these animals, it seems likely that there is an essential but low level of Fas II that is required for the proper growth and elaboration of presynaptic endings on the motor neuron dendritic arbors. It may well be that, as at the larval NMJ, this critical level of Fas II is a significant determinant of plasticity at central synapses in the fly.

\section{REFERENCES}

Bailey CH, Chen M, Keller F, Kandel ER (1992) Serotonin-mediated endocytosis of apCAM: an early step of learning-related synaptic growth in Aplysia. Science 256:645-649.

Baines RA, Bate M (1998) Electrophysiological development of central neurons in the Drosophila embryo. J Neurosci 18:4673-4683.

Baines RA, Robinson SG, Fujioka M, Jaynes JB, Bate M (1999) Postsynaptic vesicle release is essential for synaptogenesis in Drosophila. Curr Biol 9:1267-1270.

Baines RA, Uhler JP, Thompson A, Sweeney ST, Bate M (2001) Altered electrical properties in Drosophila neurons developing without synaptic transmission. J Neurosci 21:1523-1531.

Cheng Y, Endo K, Wu K, Rodan AR, Heberlein U, Davis RL (2001) Drosophila fasciclinII is required for the formation of odor memories and for normal sensitivity to alcohol. Cell 105:757-768.

Chiba A (1999) Early development of the Drosophila neuromuscular junction: a model for studying neuronal networks in development. Int Rev Neurobiol 43:1-24.

Davis GW, Goodman CS (1998) Synapse-specific control of synaptic efficacy at the terminals of a single neuron. Nature 392:82-86.

Davis GW, Schuster CM, Goodman CS (1997) Genetic analysis of the mechanisms controlling target selection: target-derived Fasciclin II regulates the pattern of synapse formation. Neuron 19:561-573.

Dityatev A, Dityateva G, Schachner M (2000) Synaptic strength as a function of post- versus presynaptic expression of the neural cell adhesion molecule NCAM. Neuron 26:207-217.

Fujioka M, Emi-Sarker Y, Yusibova GL, Goto T, Jaynes JB (1999) Analysis of an even-skipped rescue transgene reveals both composite and discrete neuronal and early blastoderm enhancers, and multi-stripe positioning by gap gene repressor gradients. Development 126:2527-2538.

Ghysen A, O'Kane C (1989) Neural enhancer-like elements as specific cell markers in Drosophila. Development 105:35-52.

Goodman CS, Shatz CJ (1993) Developmental mechanisms that generate precise patterns of neuronal connectivity. Cell 72:77-98.

Goodman CS, Davis GW, Zito K (1997) The many faces of Fasciclin II: genetic analysis reveals multiple roles for a cell adhesion molecule during the generation of neuronal specificity. Cold Spring Harb Symp Quant Biol 62:479-491.

Grenningloh G, Bieber A, Rehm J, Snow PM, Traquina Z, Hortsch M, Patel NH, Goodman CS (1990) Molecular genetics of neuronal recognition in Drosophila: evolution and function of immunoglobulin superfamily cell adhesion molecules. Cold Spring Harbor Symp Quant Biol 55:327-340.

Grenningloh G, Rehm EJ, Goodman CS (1991) Genetic analysis of growth cone guidance in Drosophila: Fasciclin II functions as a neuronal recognition molecule. Cell 67:45-57.

Haverkamp LJ (1986) Anatomical and physiological development of the Xenopus embryonic motor system in the absence of neural activity. J Neurosci 6:1338-1348.

Haverkamp LJ, Oppenheim RW (1986) Behavioral development in the absence of neural activity: effects of chronic immobilization on amphibian embryos. J Neurosci 6:1332-1337.

Hiesinger PR, Reiter C, Schau H, Fischbach K-F (1999) Neuropil pattern formation and regulation of cell adhesion molecules in Drosophila optic lobe development depend on synaptobrevin. J Neurosci 19:7548-7556.

Katz LC, Shatz CJ (1996) Synaptic activity and the construction of cortical circuits. Science 274:1133-1138.

Lin DM, Fetter RD, Kopczynski C, Grenningloh G, Goodman CS (1994) Genetic analysis of Fasciclin II in Drosophila: defasciculation, refasciculation, and altered fasciculation. Neuron 13:1055-1069.

Mayford M, Barzilai A, Keller F, Schacher S, Kandel ER (1992) Modulation of an NCAM-related adhesion molecule with long-term synaptic plasticity in Aplysia. Science 256:638-643.

Rechsteiner M (1988) Regulation of enzyme levels by proteolysis: the role of PEST regions. Adv Enzyme Regul 27:135-151.

Salvaterra PM, Kitamoto T (2001) Drosophila cholinergic neurons and processes visualized with Gal4/UAS-GFP. Gene Expr Patterns 1:73-82.

Schuster CM, Davis GW, Fetter RD, Goodman CS (1996a) Genetic dissection and functional components of synaptic plasticity. I. Fasciclin II controls synaptic stabilisation and growth. Neuron 17:641-654.

Schuster CM, Davis GW, Fetter RD, Goodman CS (1996b) Genetic dissection and functional components of synaptic plasticity. II. Fasciclin II controls presynaptic structural plasticity. Neuron 17:655-667.

Sweeney ST, Broadie K, Keane J, Niemann H, O'Kane J (1995) Targeted expression of tetanus toxin light chain in Drosophila specifically eliminates synaptic transmission and causes behavioral defects. Neuron 14:341-351.

Turrigiano GG (2000) AMPA receptors unbound: membrane cycling and synaptic plasticity. Neuron 26:5-8.

Uhler JP (2001) The development of dendritic arbors in Drosophila motoneurons, PhD thesis. University of Cambridge.

Van Vactor D, Sink H, Famborough D, Tsoo R, Goodman CS (1993) Genes that control neuromuscular specificity in Drosophila. Cell 73:1137-1153.

Zhu H, Wu F, Schacher S (1995) Changes in expression and distribution of Aplysia cell adhesion molecules can influence synapse formation and elimination in vitro. J Neurosci 15:4173-4183. 